CRYSTALLOGRAPHIC COMMUNICATIONS

ISSN 2056-9890

Received 5 November 2017

Accepted 15 November 2017

Edited by A. J. Lough, University of Toronto, Canada

Keywords: crystal structure; co-crystal salt; imidazole; picric acid.

CCDC reference: 1585713

Supporting information: this article has supporting information at journals.iucr.org/e

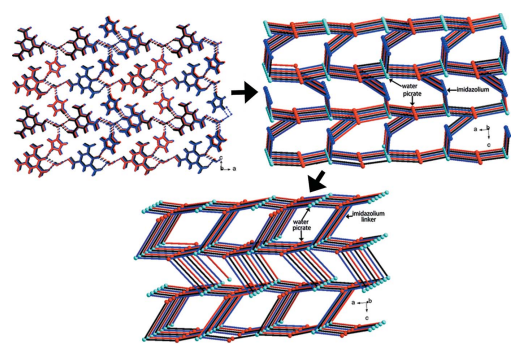

OPEN $\odot$ ACCESS

\section{Stoichiometric and polymorphic salt of imidazolium picrate monohydrate}

\author{
Ling-li Liu*
}

Renmin Hospital of Wuhan University, Wuhan 430060, People's Republic of China. *Correspondence e-mail: lingliliu573@126.com

The asymmetric unit of the title co-crystal salt, $1 \mathrm{H}$-imidazol-3-ium 2,4,6trinitrophenolate monohydrate, $\mathrm{C}_{4} \mathrm{H}_{7} \mathrm{~N}_{2}{ }^{+} \cdot \mathrm{C}_{6} \mathrm{H}_{2} \mathrm{~N}_{3} \mathrm{O}_{7}{ }^{-} \cdot \mathrm{H}_{2} \mathrm{O}$, contains one imidazolium cation, one picrate anion and one solvent water molecule of crystallization. The phenolic proton has been transferred to an imidazole $\mathrm{N}$ atom. In the crystal, the components are linked by $\mathrm{N}-\mathrm{H} \cdots \mathrm{O}$ and $\mathrm{O}-\mathrm{H} \cdots \mathrm{O}$ hydrogen bonds into a three-dimensional network which is further consolidated by weak $\mathrm{C}-\mathrm{H} \cdots \mathrm{O}$ hydrogen bonds. In addition, $\pi-\pi$ stacking interactions occur between pairs of imidazolium cations and picrate anions. If only the classical $\mathrm{N}-\mathrm{H} \cdots \mathrm{O}$ and $\mathrm{O}-\mathrm{H} \cdots \mathrm{O}$ hydrogen bonds are considered, the component ions are linked into a three-dimensional threefold interpenetrating network of the topological type utp [or $(10,3)-d]$. Hirshfeld surface analysis indicates the crystal structure is mainly stabilized by $\mathrm{H} \cdots \mathrm{O}$ contacts of the hydrogen bonds.

\section{Chemical context}

Co-crystallization, which is the crystallization of more than one solid component into a new compound, is widely involved in the research fields of active pharmaceuticals (Aitipamula et al., 2015; Weyna et al., 2012; Robinson, 2010; Arenas-García et al., 2010) and crystal engineering (Manoj et al., 2014). Imidazole is an often used intermediate in pharmaceutical and chemical synthesis. Its crystallization characteristics can facilitate organic synthesis and theoretical prediction. Picric acid is a strong organic proton-donating reagent which can favor the crystallization of some basic organic complexes. By controlling one specific crystallization condition such as solvent, temperature, pressure or molar ratio of the raw materials, some polymorphs can be obtained with the same ingredients. For instance, two imidazolium picrate co-crystal salts have been reported that were crystallized from chloroform (Soriano-García et al., 1990) or dry acetonitrile (MorenoFuquen et al., 2011). The crystal packing in these two analogs is completely different because of their different stoichiometric compositions. In order to further research the factors affecting this crystallization process, the crystallization solvent has been adjusted to be methanol (95\%). Interestingly, some yellow needle-shaped crystals were obtained after two days on the side of the vessel and when the solvent had almost evaporated, several yellow block-shaped crystals formed at the bottom of the vessel (Fig. $1 a$ and $1 b$ ). The results of X-ray diffraction indicates that the structure of the block-shaped crystals is the same as that reported by Moreno-Fuquen et al. (2011). Herein, the crystal structure of the needle-shaped crystals is reported. 


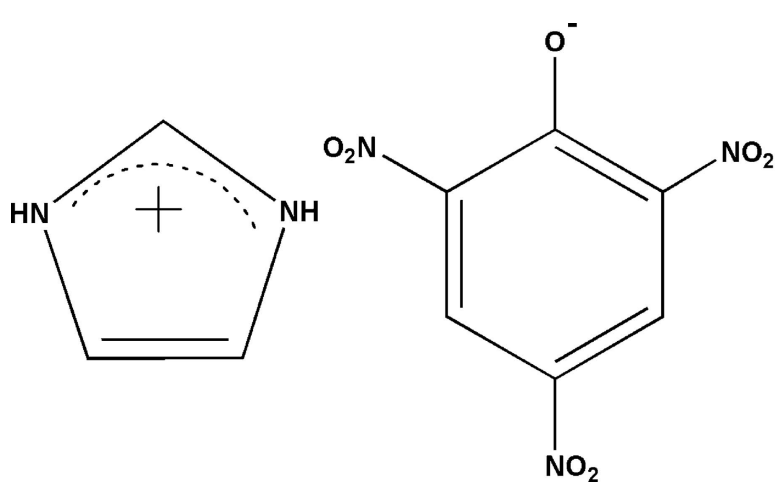

\section{Structural commentary}

The asymmetric unit of the title compound (I) contains one imidazolium cation, a picrate anion and one solvent water molecule of crystallization (Fig. 2). The phenolic proton has been transferred to a imidazole nitrogen atom, forming the solvated 1:1 co-crystal salt. In the picrate anion of $(\mathrm{I})$, the $\mathrm{C}-$ $\mathrm{O}_{\text {phenol }}$ bond distance is 1.250 (2) $\AA$, which is shorter by $c a$ $0.08 \AA$ than the value of 1.33 (2) $\AA$ in the protonated species (Bertolasi et al., 2011). Also, the two neighboring $\mathrm{C}-\mathrm{C}$ bonds [1.447 (3) $\AA$ for $\mathrm{C} 1-\mathrm{C} 2$ and $\mathrm{C} 1-\mathrm{C} 6]$, are significantly different from those in a benzene ring with delocalized $\mathrm{C} \cdots \mathrm{C}$ bonds. The $\mathrm{C} 2-\mathrm{C} 1-\mathrm{C} 6$ angle $\left[110.56(16)^{\circ}\right]$ is smaller by $c a$ $11.3^{\circ}$ than the averaged value of the other five ring inner angles $\left[121.8(1)^{\circ}\right]$. This deviation of bonds and angles can mainly be attributed to the electron-withdrawing effects of the three nitro groups, which can delocalize the negative charge on the phenolate $\mathrm{O} 1$ atom over the whole $\pi$-conjugated system. The nitro groups, N1/O2/O3, N2/O4/O5 and N3/O6/ $\mathrm{O} 7$, are twisted from the central benzene ring by dihedral angles of $43.3(3), 4.2(3)$ and $48.5(3)^{\circ}$, respectively. In the imidazolium cation, the $\mathrm{C} 7-\mathrm{N} 4[1.329(3) \AA]$ and $\mathrm{C} 7-\mathrm{N} 5$ [1.331 (3) $\AA$ ] bond distances are the same due to the delocalizing effect and similar to those observed in other co-crystal salts (Soriano-García et al., 1990; Moreno-Fuquen et al., 2011).

\section{Supramolecular features}

In the crystal of (I), the three components are linked into a three-dimensional network by $\mathrm{N}-\mathrm{H} \cdots \mathrm{O}$ and $\mathrm{O}-\mathrm{H} \cdots \mathrm{O}$

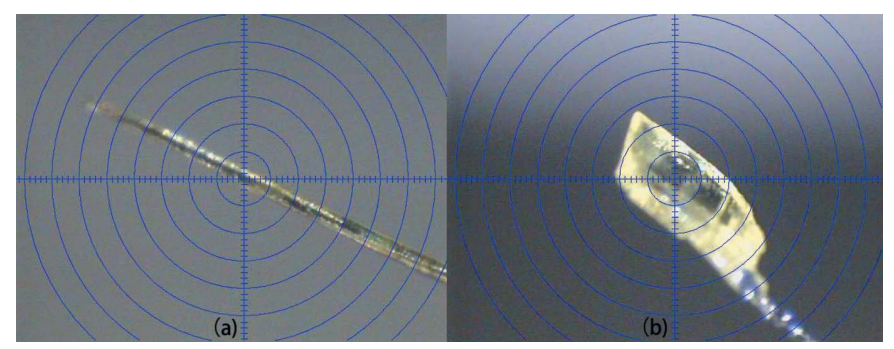

Figure 1

The morphologies of the two molecular salts: needle $(a)$ of (I) and block (b) of the crystal structure reported by Moreno-Fuquen et al. (2011).
Table 1

Hydrogen-bond geometry $\left(\AA{ }^{\circ}\right)$.

\begin{tabular}{|c|c|c|c|c|}
\hline$D-\mathrm{H} \cdots A$ & $D-\mathrm{H}$ & $\mathrm{H} \cdots A$ & $D \cdots A$ & $D-\mathrm{H} \cdots A$ \\
\hline $\mathrm{N} 4-\mathrm{H} 4 \cdots \mathrm{O} 8^{\mathrm{i}}$ & $0.93(3)$ & $1.83(3)$ & $2.763(3)$ & $172(3)$ \\
\hline $\mathrm{N} 5-\mathrm{H} 5 A \cdots \mathrm{O} 1^{\mathrm{ii}}$ & $0.89(3)$ & 1.94 (3) & $2.812(2)$ & $165(3)$ \\
\hline $\mathrm{N} 5-\mathrm{H} 5 A \cdots \mathrm{O} 3^{\mathrm{ii}}$ & $0.89(3)$ & $2.46(3)$ & $2.956(3)$ & $116(2)$ \\
\hline $\mathrm{O} 8-\mathrm{H} 8 A \cdots \mathrm{O} 4^{\mathrm{iii}}$ & $0.82(4)$ & $2.29(4)$ & $3.034(2)$ & $151(3)$ \\
\hline $\mathrm{O} 8-\mathrm{H} 8 B \cdots \mathrm{O} 1^{\mathrm{ii}}$ & $0.88(4)$ & $2.04(4)$ & $2.899(2)$ & $165(3)$ \\
\hline $\mathrm{O} 8-\mathrm{H} 8 B \cdots \mathrm{O} 6^{\mathrm{ii}}$ & $0.88(4)$ & $2.44(4)$ & $2.943(3)$ & $117(3)$ \\
\hline $\mathrm{C} 5-\mathrm{H} 5 \cdots \mathrm{O} 2^{\mathrm{iv}}$ & 0.95 & 2.49 & $3.383(3)$ & 157 \\
\hline $\mathrm{C} 7-\mathrm{H} 7 \cdots \mathrm{O}^{\mathrm{v}}$ & 0.95 & 2.37 & $3.187(3)$ & 144 \\
\hline $\mathrm{C} 8-\mathrm{H} 8 \cdots \mathrm{O} 4^{\mathrm{vi}}$ & 0.95 & 2.44 & $3.188(3)$ & 135 \\
\hline $\mathrm{C} 8-\mathrm{H} 8 \cdots \mathrm{O} 8$ & 0.95 & 2.53 & $3.255(3)$ & 133 \\
\hline $\mathrm{C} 9-\mathrm{H} 9 \cdots \mathrm{O}^{\mathrm{vii}}$ & 0.95 & 2.48 & $3.349(3)$ & 152 \\
\hline
\end{tabular}

Symmetry codes: (i) $-x+1,-y+1, z+\frac{1}{2}$; (ii) $x, y+1, z$; (iii) $x-\frac{1}{2},-y+\frac{3}{2}, z$; (iv) $-x+\frac{3}{2}, y+\frac{1}{2}, z-\frac{1}{2} ; \quad$ (v) $\quad-x+\frac{3}{2}, y+\frac{1}{2}, z+\frac{1}{2} ; \quad$ (vi) $\quad x-\frac{1}{2},-y+\frac{1}{2}, z ; \quad$ (vii) $-x+1,-y, z+\frac{1}{2}$.

hydrogen bonds (Table 1, Fig. 3). In order to understand the structure simply, we can analyze it in the terms below. Firstly, the imidazolium cations, picrate anions and water molecules are linked by each three $\mathrm{N}-\mathrm{H} \cdots \mathrm{O}$ and three $\mathrm{O}-\mathrm{H} \cdots \mathrm{O}$ hydrogen bonds, forming a three-dimensional framework structure (Fig. 3; Spek, 2003, 2009). It is worthy mentioning that if both the water molecule and the picrate anion are regarded as 3-connected nodes by hydrogen-bonding and the imidazole cation as a 2-connected node, then the threedimensional framework can be viewed topologically as a 3connected utp network with a short Schläfli symbol of $\left(10^{3}\right)-d$ (Blatov et al., 2014; Baburin \& Blatov, 2007) (Fig. 3). Secondly, the three-dimensional hydrogen-bonded framework is consolidated by $\pi-\pi$ interactions between pairs of imidazolium cations and picrate anions, both with centroid-to-centroid distances of 3.553 (4) $\AA$, and weak intermolecular $\mathrm{C}-\mathrm{H} \cdots \mathrm{O}$ interactions (Table 1). It should be mentioned that the short $\mathrm{O} 2 \cdots \mathrm{O} 7\left(\frac{3}{2}-x, y-\frac{1}{2}, \frac{1}{2}+z\right)$ contact of 2.837 (4) $\AA$ may be the result of an inclined $\mathrm{NO}_{2} \cdots \pi\left(\mathrm{NO}_{2}\right)$ interaction (Daszkiewicz, 2013)

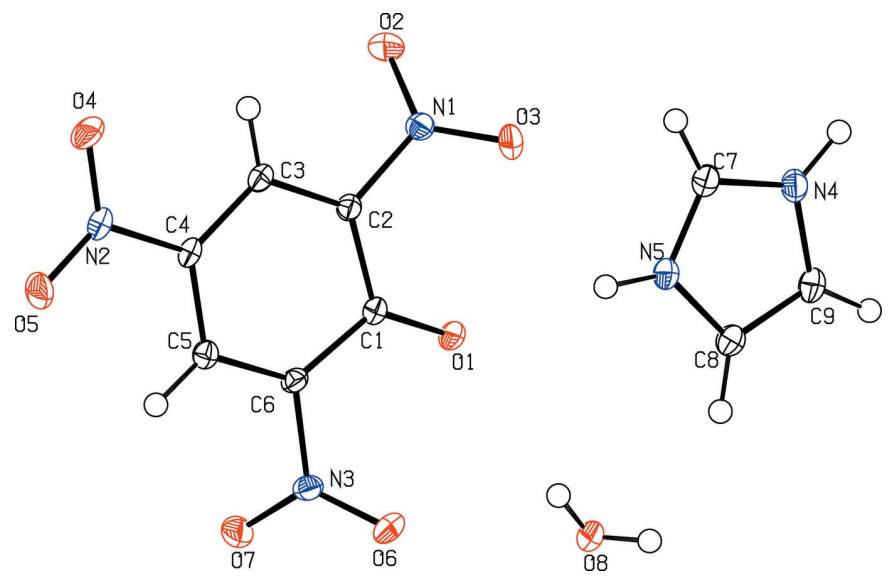

Figure 2

Molecular structure of (I), showing the atom-numbering scheme. Displacement ellipsoids are drawn at the $50 \%$ probability level. 


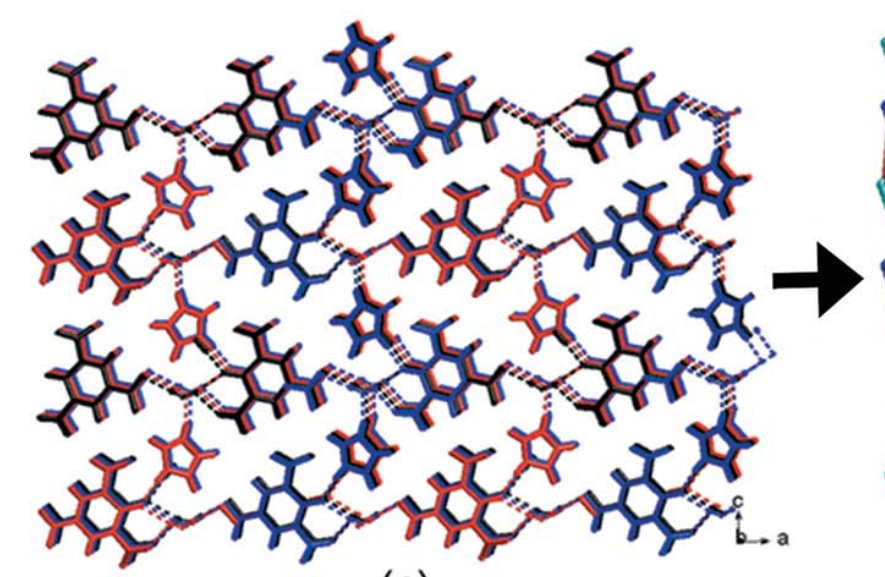

(a)

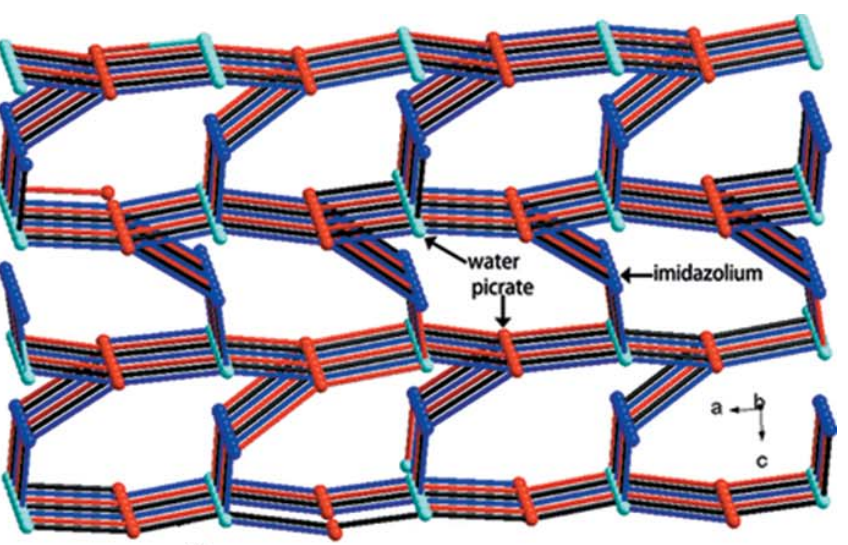

(b)

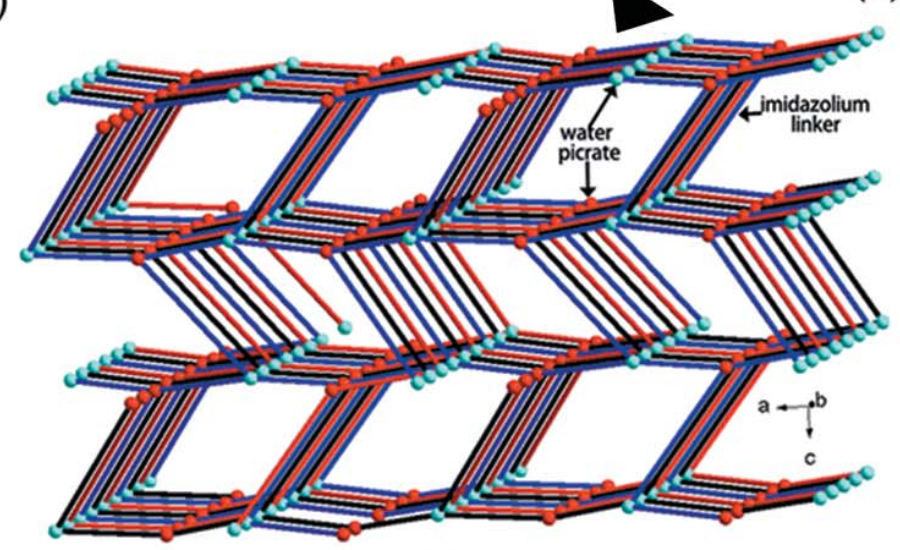

(c)

Figure 3

Part of the crystal structure of (I), showing $(a)$ the formation of the three-dimensional hydrogen-bonded network by $\mathrm{N}-\mathrm{H} \cdots \mathrm{O}$ and $\mathrm{O}-\mathrm{H} \cdots \mathrm{O}$ hydrogen bonds as dashed lines, $(b)$ the simplified network when picrate, water and imidazolium ions are considered as 3-, 3- and 2-connected nodes, respectively, and (c) the simplified utp network.

\section{Hirshfeld surface analysis}

An alternative way to asses the intermolecular interactions quantitatively around one specific molecule is through Hirshfeld surface analysis (Wolff et al., 2012; McKinnon et al., 2004). The Hirshfeld surface can define the environment of each crystallographically independent molecule within a crystal. Fingerprint plots (Fig. 4) and show that for the picrate anion $55.2 \%$ and $8.8 \%$ of the area is concerned with the $\mathrm{O} \cdots \mathrm{H}$ (hydrogen-bonding) and $\mathrm{C} \cdot \mathrm{C}(\pi-\pi$ interaction) contacts, respectively. In the imidazole cation, $51.5 \%$ and $9.7 \%$ of the area is concerned with the the $\mathrm{O} \cdots \mathrm{H}$ (hydrogenbonding) and $\mathrm{C} \cdots \mathrm{C} / \mathrm{N}(\pi-\pi$ interaction) contacts, respectively. This quantitative analysis of the intermolecular interactions again shows that the three-dimensional network is defined mainly by hydrogen bonds.

\section{Database survey}

A search of the Cambridge Structural Database (CSD version 5.37 plus one update, Groom et al., 2016) indicates some analogs have been reported, viz. BEZGEU (Dhanabal et al., 2013), QAKYOS (Dutkiewicz et al., 2011), QAKGUG (Moreno-Fuquen et al., 2011) and SEZREU (Soriano-García
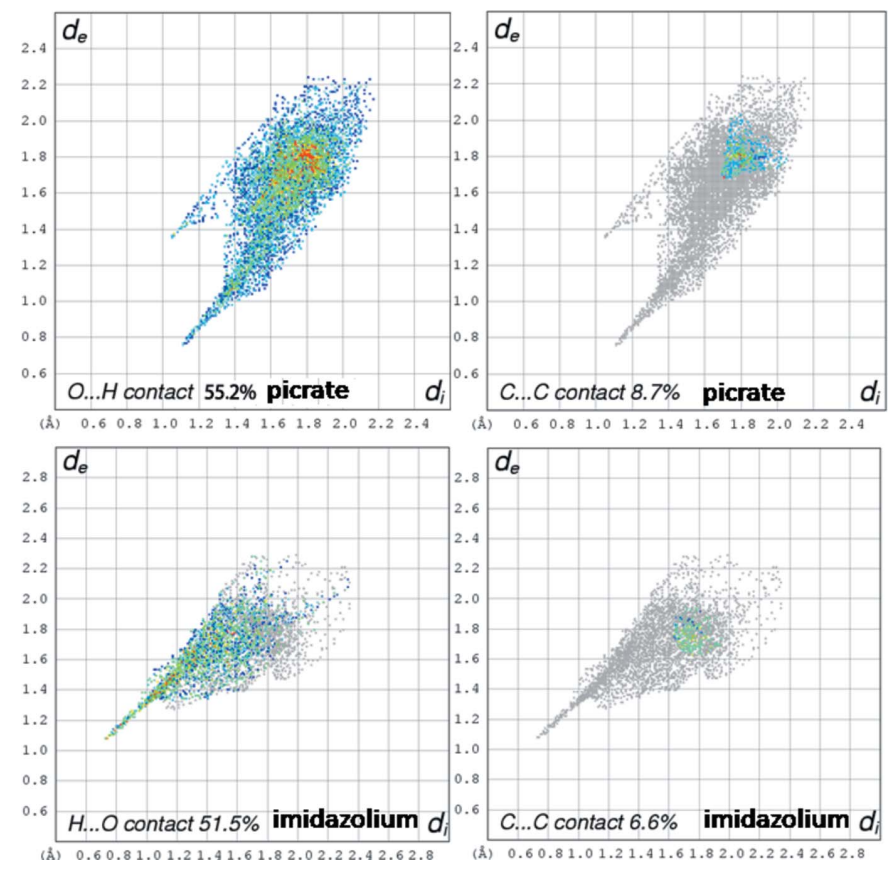

Figure 4

Fingerprint plots of co-crystal salt (I) showing the percentage of the $\mathrm{O} \cdots \mathrm{H}$ and $\mathrm{C} \cdots \mathrm{C}$ contacts around the environment of picrate and imidazolium ions. 
et al., 1990). A structural comparison between these compounds indicates that the two nitrogen atoms in the imidazolium cations are preferably hydrogen-bonded to the picrate anions, in which they can be in a bifurcated or a linear mode. For instance, in the 1:1 organic salt imidazolium picrate (QAKGUG; Moreno-Fuquen et al., 2011), the imidazole N7 atom is linearly hydrogen-bonded to the phenolate oxygen atom O1. However, in the 1:2 salt (SEZREU; Soriano-García et al., 1990), the imidazole N1 atom is involved in bifuracted hydrogen bonding to the phenolate $\mathrm{O} 1$ and nitro $\mathrm{O} 2$ atoms. Both of these compounds crystallize in orthorhombic space groups ( $P b c a$ or $\left.P 2_{1} 2_{1} 2_{1}\right)$. Further research about polymorphism in this system is being carried out in our lab.

\section{Synthesis and crystallization}

All the reagents and solvents were used as obtained without further purification. Equivalent molar amounts of imidazole $(1.0 \mathrm{mmol}, 68.0 \mathrm{mg})$ and picric acid $(1.0 \mathrm{mmol}, 229.0 \mathrm{mg})$ were dissolved in $95 \%$ methanol $(40.0 \mathrm{ml})$. The mixture was stirred for half an hour at ambient temperature and then filtered. The resulting yellow solution was kept in air for two weeks. Yellow needle-shaped crystals of (I) suitable for single-crystal X-ray diffraction analysis formed on the side of the vessel after two days. The crystals were separated manually (yield: $75 \%, c a$ $0.24 \mathrm{~g})$.

\section{Refinement}

Crystal data, data collection and structure refinement details of compound (I) are summarized in Table 2. $\mathrm{H}$ atoms bonded to $\mathrm{C}$ atoms were positioned geometrically with $\mathrm{C}-\mathrm{H}=0.93 \AA$ (aromatic) and refined in riding mode $\left[U_{\text {iso }}(\mathrm{H})=1.2 U_{\text {eq }}(\mathrm{C})\right]$. $\mathrm{H}$ atoms bonded to $\mathrm{N}$ and $\mathrm{O}$ atoms were found in differenceFourier maps and refined freely with constraints of $U_{\text {iso }}(\mathrm{H})=$ $1.2 U_{\mathrm{eq}}(\mathrm{N})$ or $1.5 U_{\mathrm{eq}}(\mathrm{N})$.

\section{Funding information}

The author thanks Renmin Hospital of Wuhan University for the financial support.

\section{References}

Aitipamula, S., Mapp, L. K., Wong, A. B. H., Chow, P. S. \& Tan, R. B. H. (2015). CrystEngComm, 17, 9323-9335.

Arenas-García, J. I., Herrera-Ruiz, D., Mondragón-Vásquez, K., Morales-Rojas, H. \& Höpfl, H. (2010). Cryst. Growth Des. 10, 37323742.

Baburin, I. A. \& Blatov, V. A. (2007). Acta Cryst. B63, 791-802.

Bertolasi, V., Gilli, P. \& Gilli, G. (2011). Cryst. Growth Des. 11, $2724-$ 2735 .

Blatov, V. A., Shevchenko, A. P. \& Proserpio, D. M. (2014). Cryst. Growth Des. 14, 3576-3586.

Brandenburg, K. (2006). DIAMOND. Crystal Impact GbR, Bonn, Germany.
Table 2

Experimental details.

\begin{tabular}{|c|c|}
\hline \multicolumn{2}{|l|}{ Crystal data } \\
\hline Chemical formula & $\mathrm{C}_{3} \mathrm{H}_{5} \mathrm{~N}_{2}{ }^{+} \cdot \mathrm{C}_{6} \mathrm{H}_{2} \mathrm{~N}_{3} \mathrm{O}_{7}^{-} \cdot \mathrm{H}_{2} \mathrm{O}$ \\
\hline$M_{\mathrm{r}}$ & 315.21 \\
\hline Crystal system, space group & Orthorhombic, Pna2 1 \\
\hline Temperature (K) & 100 \\
\hline$a, b, c(\AA)$ & $21.577(11), 3.5533(18), 16.096(8)$ \\
\hline$V\left(\AA^{3}\right)$ & $1234.0(11)$ \\
\hline$Z$ & 4 \\
\hline Radiation type & Мо $K \alpha$ \\
\hline$\mu\left(\mathrm{mm}^{-1}\right)$ & 0.15 \\
\hline Crystal size $(\mathrm{mm})$ & $0.40 \times 0.04 \times 0.02$ \\
\hline \multicolumn{2}{|l|}{ Data collection } \\
\hline Diffractometer & Bruker APEXII CCD \\
\hline Absorption correction & $\begin{array}{l}\text { Multi-scan (SADABS; Bruker, } \\
\text { 2001) }\end{array}$ \\
\hline$T_{\min }, T_{\max }$ & $0.937,0.997$ \\
\hline $\begin{array}{l}\text { No. of measured, independent and } \\
\text { observed }[I>2 \sigma(I)] \text { reflections }\end{array}$ & $11664,3837,3367$ \\
\hline$R_{\text {int }}$ & 0.038 \\
\hline$(\sin \theta / \lambda)_{\max }\left(\AA^{-1}\right)$ & 0.724 \\
\hline \multicolumn{2}{|l|}{ Refinement } \\
\hline$R\left[F^{2}>2 \sigma\left(F^{2}\right)\right], w R\left(F^{2}\right), S$ & $0.035,0.086,1.04$ \\
\hline No. of reflections & 3837 \\
\hline No. of parameters & 211 \\
\hline No. of restraints & 1 \\
\hline $\mathrm{H}$-atom treatment & $\begin{array}{l}\mathrm{H} \text { atoms treated by a mixture of } \\
\text { independent and constrained } \\
\text { refinement }\end{array}$ \\
\hline$\Delta \rho_{\max }, \Delta \rho_{\min }\left(\mathrm{e} \AA^{-3}\right)$ & $0.35,-0.22$ \\
\hline
\end{tabular}

Computer programs: APEX2 and SAINT (Bruker, 2001), SHELXS97 and SHELXTL (Sheldrick, 2008), SHELXL2014 (Sheldrick, 2015) and DIAMOND (Brandenburg, 2006)

Bruker (2001). APEX2, SAINT \& SADABS . Bruker AXS, Inc., Madison, Wisconsin, USA.

Daszkiewicz, M. (2013). CrystEngComm, 15, 10427-10430.

Dhanabal, T., Sethuram, M., Amirthaganesan, G. \& Das, S. K. (2013). J. Mol. Struct. 1045, 112-123.

Dutkiewicz, G., Samshuddin, S., Narayana, B., Yathirajan, H. S. \& Kubicki, M. (2011). Acta Cryst. E67, o235.

Groom, C. R., Bruno, I. J., Lightfoot, M. P. \& Ward, S. C. (2016). Acta Cryst. B72, 171-179.

Manoj, K., Tamura, R., Takahashi, H. \& Tsue, H. (2014). CrystEngComm, 16, 5811-5819.

McKinnon, J. J., Spackman, M. A. \& Mitchell, A. S. (2004). Acta Cryst. B60, 627-668.

Moreno-Fuquen, R., De Almeida Santos, R. \& Aguirre, L. (2011). Acta Cryst. E67, o139.

Robinson, D. I. (2010). Org. Process Res. Dev. 14, 946-959.

Sheldrick, G. M. (2008). Acta Cryst. A64, 112-122.

Sheldrick, G. M. (2015). Acta Cryst. C71, 3-8.

Soriano-García, M., Schatz-Levine, M., Toscano, R. A. \& Villena Iribe, R. (1990). Acta Cryst. C46, 1556-1558.

Spek, A. L. (2003). J. Appl. Cryst. 36, 7-13.

Spek, A. L. (2009). Acta Cryst. D65, 148-155.

Weyna, D. R., Cheney, M. L., Shan, N., Hanna, M., Wojtas, Ł. \& Zaworotko, M. J. (2012). CrystEngComm, 14, 2377-2380.

Wolff, S. K., Grimwood, D. J., McKinnon, J. J., Turner, M. J., Jayatilaka, D. \& Spackman, M. A. (2012). Crystal Explorer. University of Western Australia. 


\section{supporting information}

Acta Cryst. (2017). E73, 1885-1888 [https://doi.org/10.1107/S2056989017016401]

\section{Stoichiometric and polymorphic salt of imidazolium picrate monohydrate}

\section{Ling-li Liu}

\section{Computing details}

Data collection: APEX2 (Bruker, 2001); cell refinement: SAINT (Bruker, 2001); data reduction: SAINT (Bruker, 2001); program(s) used to solve structure: SHELXS97 (Sheldrick, 2008); program(s) used to refine structure: SHELXL2014 (Sheldrick, 2015); molecular graphics: SHELXTL (Sheldrick, 2008) and DIAMOND (Brandenburg, 2006); software used to prepare material for publication: SHELXTL (Sheldrick, 2008).

1H-Imidazol-3-ium 2,4,6-trinitrophenolate monohydrate

Crystal data

$\mathrm{C}_{3} \mathrm{H}_{5} \mathrm{~N}_{2}{ }^{+} \cdot \mathrm{C}_{6} \mathrm{H}_{2} \mathrm{~N}_{3} \mathrm{O}_{7} \cdot \cdot \mathrm{H}_{2} \mathrm{O}$

$M_{r}=315.21$

Orthorhombic, Pna2

$a=21.577(11) \AA$

$b=3.5533(18) \AA$

$c=16.096(8) \AA$

$V=1234.0(11) \AA^{3}$

$Z=4$

$F(000)=648$

\section{Data collection}

Bruker APEXII CCD diffractometer

$\varphi$ and $\omega$ scans

Absorption correction: multi-scan

(SADABS; Bruker, 2001)

$T_{\min }=0.937, T_{\max }=0.997$

11664 measured reflections

\section{Refinement}

Refinement on $F^{2}$

Least-squares matrix: full

$R\left[F^{2}>2 \sigma\left(F^{2}\right)\right]=0.035$

$w R\left(F^{2}\right)=0.086$

$S=1.04$

3837 reflections

211 parameters

1 restraint

Hydrogen site location: mixed
$D_{\mathrm{x}}=1.697 \mathrm{Mg} \mathrm{m}^{-3}$

Mo $K \alpha$ radiation, $\lambda=0.71073 \AA$

Cell parameters from 4015 reflections

$\theta=2.3-31.2^{\circ}$

$\mu=0.15 \mathrm{~mm}^{-1}$

$T=100 \mathrm{~K}$

Needle, yellow

$0.40 \times 0.04 \times 0.02 \mathrm{~mm}$

3837 independent reflections

3367 reflections with $I>2 \sigma(I)$

$R_{\text {int }}=0.038$

$\theta_{\text {max }}=31.0^{\circ}, \theta_{\min }=1.9^{\circ}$

$h=-28 \rightarrow 30$

$k=-4 \rightarrow 5$

$l=-23 \rightarrow 22$

$\mathrm{H}$ atoms treated by a mixture of independent and constrained refinement

$w=1 /\left[\sigma^{2}\left(F_{\mathrm{o}}^{2}\right)+(0.0494 P)^{2}+0.0144 P\right]$ where $P=\left(F_{\mathrm{o}}^{2}+2 F_{\mathrm{c}}^{2}\right) / 3$

$(\Delta / \sigma)_{\max }<0.001$

$\Delta \rho_{\max }=0.35$ e $\AA^{-3}$

$\Delta \rho_{\min }=-0.22$ e $\AA^{-3}$

Absolute structure: Flack $x$ determined using 1456 quotients $\left[\left(I^{+}\right)-\left(I^{-}\right)\right] /\left[\left(I^{+}\right)+\left(I^{\prime}\right)\right]$ (Parsons et al., 2013)

Absolute structure parameter: 0.4 (5) 


\section{Special details}

Geometry. All esds (except the esd in the dihedral angle between two 1.s. planes) are estimated using the full covariance matrix. The cell esds are taken into account individually in the estimation of esds in distances, angles and torsion angles; correlations between esds in cell parameters are only used when they are defined by crystal symmetry. An approximate (isotropic) treatment of cell esds is used for estimating esds involving l.s. planes.

Fractional atomic coordinates and isotropic or equivalent isotropic displacement parameters $\left(\AA^{2}\right)$

\begin{tabular}{|c|c|c|c|c|}
\hline & $x$ & $y$ & $z$ & $U_{\text {iso }} * / U_{\text {eq }}$ \\
\hline $\mathrm{C} 1$ & $0.67480(9)$ & $-0.0182(6)$ & $0.64727(12)$ & $0.0122(3)$ \\
\hline $\mathrm{C} 2$ & $0.72241(9)$ & $0.0454(6)$ & $0.70899(11)$ & $0.0125(4)$ \\
\hline $\mathrm{C} 3$ & $0.78154(9)$ & $0.1698(6)$ & $0.69303(11)$ & $0.0131(4)$ \\
\hline $\mathrm{H} 3$ & 0.8106 & 0.2010 & 0.7368 & $0.016^{*}$ \\
\hline $\mathrm{C} 4$ & $0.79782(9)$ & $0.2488(6)$ & $0.61128(12)$ & $0.0128(3)$ \\
\hline $\mathrm{C} 5$ & $0.75625(8)$ & $0.1917(6)$ & $0.54609(12)$ & $0.0133(4)$ \\
\hline H5 & 0.7681 & 0.2377 & 0.4902 & $0.016^{*}$ \\
\hline C6 & $0.69788(9)$ & $0.0674(6)$ & $0.56500(12)$ & $0.0125(3)$ \\
\hline $\mathrm{C} 8$ & $0.48877(9)$ & $0.4718(7)$ & $0.77357(13)$ & $0.0172(4)$ \\
\hline $\mathrm{H} 8$ & 0.4715 & 0.4535 & 0.7194 & $0.021^{*}$ \\
\hline C9 & $0.46050(9)$ & $0.3770(7)$ & $0.84591(13)$ & $0.0181(4)$ \\
\hline H9 & 0.4197 & 0.2809 & 0.8522 & $0.022 *$ \\
\hline N1 & $0.70790(8)$ & $-0.0342(5)$ & $0.79547(11)$ & $0.0143(3)$ \\
\hline $\mathrm{N} 2$ & $0.85824(8)$ & $0.4007(5)$ & $0.59405(11)$ & $0.0150(3)$ \\
\hline N3 & $0.65576(8)$ & $0.0095(5)$ & $0.49511(11)$ & $0.0149(3)$ \\
\hline $\mathrm{C} 7$ & $0.55452(10)$ & $0.5801(7)$ & $0.87530(13)$ & $0.0172(4)$ \\
\hline H7 & 0.5908 & 0.6495 & 0.9049 & $0.021^{*}$ \\
\hline N4 & $0.50257(9)$ & $0.4472(6)$ & $0.90866(12)$ & $0.0170(3)$ \\
\hline $\mathrm{H} 4$ & $0.4957(14)$ & $0.398(9)$ & $0.9649(19)$ & $0.020^{*}$ \\
\hline N5 & $0.54696(8)$ & $0.5991(5)$ & $0.79335(11)$ & $0.0157(3)$ \\
\hline $\mathrm{H} 5 \mathrm{~A}$ & $0.5763(13)$ & $0.682(8)$ & $0.7586(18)$ & $0.019^{*}$ \\
\hline $\mathrm{O} 1$ & $0.62270(6)$ & $-0.1576(5)$ & $0.66176(9)$ & $0.0156(3)$ \\
\hline $\mathrm{O} 4$ & $0.89440(7)$ & $0.4486(5)$ & $0.65267(10)$ & $0.0226(3)$ \\
\hline O5 & $0.87159(7)$ & $0.4829(5)$ & $0.52189(10)$ & $0.0226(4)$ \\
\hline $\mathrm{O} 8$ & $0.50725(7)$ & $0.6717(6)$ & $0.57776(10)$ & $0.0214(3)$ \\
\hline H8A & $0.4849(16)$ & $0.841(10)$ & $0.594(2)$ & $0.032^{*}$ \\
\hline $\mathrm{H} 8 \mathrm{~B}$ & $0.5441(17)$ & $0.745(10)$ & $0.595(2)$ & $0.032^{*}$ \\
\hline $\mathrm{O} 2$ & $0.74665(8)$ & $-0.1992(5)$ & $0.83731(10)$ & $0.0227(4)$ \\
\hline $\mathrm{O} 3$ & $0.65759(7)$ & $0.0718(5)$ & $0.82223(10)$ & $0.0219(3)$ \\
\hline O6 & $0.60348(7)$ & $0.1417(5)$ & $0.50023(10)$ & $0.0222(4)$ \\
\hline $\mathrm{O} 7$ & $0.67538(7)$ & $-0.1656(5)$ & $0.43500(9)$ & $0.0211(3)$ \\
\hline
\end{tabular}

Atomic displacement parameters $\left(\AA^{2}\right)$

\begin{tabular}{lllllll}
\hline & $U^{11}$ & $U^{22}$ & $U^{33}$ & $U^{12}$ & $U^{13}$ & $U^{23}$ \\
\hline C1 & $0.0097(7)$ & $0.0125(9)$ & $0.0143(8)$ & $0.0002(6)$ & $0.0006(6)$ & $0.0003(7)$ \\
C2 & $0.0109(8)$ & $0.0138(10)$ & $0.0127(8)$ & $0.0000(7)$ & $0.0006(6)$ & $0.0008(7)$ \\
C3 & $0.0099(8)$ & $0.0138(10)$ & $0.0157(8)$ & $0.0014(7)$ & $-0.0005(6)$ & $-0.0006(7)$ \\
C4 & $0.0085(7)$ & $0.0128(9)$ & $0.0171(8)$ & $-0.0001(6)$ & $0.0008(6)$ & $-0.0002(6)$
\end{tabular}


supporting information

$\begin{array}{lllllll}\text { C5 } & 0.0115(8) & 0.0135(10) & 0.0148(8) & -0.0003(7) & 0.0010(6) & 0.0006(7) \\ \text { C6 } & 0.0101(8) & 0.0142(10) & 0.0134(8) & -0.0012(7) & -0.0020(6) & 0.0002(7) \\ \text { C8 } & 0.0135(9) & 0.0198(11) & 0.0182(9) & -0.0029(7) & 0.0000(7) & -0.0001(8) \\ \text { C9 } & 0.0125(8) & 0.0203(11) & 0.0215(9) & -0.0020(8) & 0.0028(7) & 0.0004(8) \\ \text { N1 } & 0.0133(8) & 0.0159(9) & 0.0138(7) & -0.0034(6) & -0.0006(6) & -0.0001(6) \\ \text { N2 } & 0.0097(7) & 0.0151(9) & 0.0203(8) & 0.0000(6) & 0.0013(6) & 0.0000(7) \\ \text { N3 } & 0.0129(8) & 0.0181(9) & 0.0136(7) & -0.0029(6) & -0.0025(5) & 0.0031(6) \\ \text { C7 } & 0.0132(9) & 0.0198(11) & 0.0185(9) & 0.0008(7) & 0.0013(7) & -0.0004(8) \\ \text { N4 } & 0.0143(7) & 0.0193(9) & 0.0176(8) & 0.0004(7) & 0.0034(6) & 0.0004(7) \\ \text { N5 } & 0.0122(8) & 0.0184(9) & 0.0163(7) & -0.0012(6) & 0.0021(6) & 0.0006(7) \\ \text { O1 } & 0.0095(6) & 0.0210(8) & 0.0163(6) & -0.0035(5) & -0.0002(5) & 0.0011(6) \\ \text { O4 } & 0.0112(6) & 0.0316(10) & 0.0248(7) & -0.0045(6) & -0.0036(6) & 0.0025(7) \\ \text { O5 } & 0.0166(7) & 0.0318(10) & 0.0195(7) & -0.0063(6) & 0.0057(6) & -0.0003(6) \\ \text { O8 } & 0.0122(7) & 0.0340(10) & 0.0181(7) & 0.0016(7) & -0.0005(5) & -0.0032(7) \\ \text { O2 } & 0.0228(8) & 0.0294(10) & 0.0158(7) & 0.0039(7) & -0.0032(6) & 0.0040(7) \\ \text { O3 } & 0.0141(7) & 0.0333(10) & 0.0182(7) & -0.0005(6) & 0.0056(6) & -0.0026(6) \\ \text { O6 } & 0.0124(7) & 0.0318(10) & 0.0224(7) & 0.0042(6) & -0.0030(5) & 0.0031(7) \\ \text { O7 } & 0.0187(7) & 0.0287(10) & 0.0159(6) & -0.0031(6) & -0.0002(6) & -0.0056(6)\end{array}$

Geometric parameters $\left(\AA,{ }^{\circ}\right)$

\begin{tabular}{|c|c|c|c|}
\hline $\mathrm{C} 1-\mathrm{O} 1$ & $1.250(2)$ & $\mathrm{C} 9-\mathrm{N} 4$ & $1.381(3)$ \\
\hline $\mathrm{C} 1-\mathrm{C} 2$ & $1.447(3)$ & C9-H9 & 0.9500 \\
\hline $\mathrm{C} 1-\mathrm{C} 6$ & $1.447(3)$ & $\mathrm{N} 1-\mathrm{O} 2$ & $1.223(2)$ \\
\hline $\mathrm{C} 2-\mathrm{C} 3$ & $1.374(3)$ & $\mathrm{N} 1-\mathrm{O} 3$ & $1.227(2)$ \\
\hline $\mathrm{C} 2-\mathrm{N} 1$ & $1.455(3)$ & $\mathrm{N} 2-\mathrm{O} 5$ & $1.232(2)$ \\
\hline $\mathrm{C} 3-\mathrm{C} 4$ & $1.391(3)$ & $\mathrm{N} 2-\mathrm{O} 4$ & $1.236(2)$ \\
\hline $\mathrm{C} 3-\mathrm{H} 3$ & 0.9500 & N3-O6 & $1.225(2)$ \\
\hline $\mathrm{C} 4-\mathrm{C} 5$ & $1.395(3)$ & $\mathrm{N} 3-\mathrm{O} 7$ & $1.226(3)$ \\
\hline $\mathrm{C} 4-\mathrm{N} 2$ & $1.438(3)$ & $\mathrm{C} 7-\mathrm{N} 4$ & $1.329(3)$ \\
\hline $\mathrm{C} 5-\mathrm{C} 6$ & $1.369(3)$ & $\mathrm{C} 7-\mathrm{N} 5$ & $1.331(3)$ \\
\hline $\mathrm{C} 5-\mathrm{H} 5$ & 0.9500 & $\mathrm{C} 7-\mathrm{H} 7$ & 0.9500 \\
\hline $\mathrm{C} 6-\mathrm{N} 3$ & $1.461(3)$ & $\mathrm{N} 4-\mathrm{H} 4$ & $0.93(3)$ \\
\hline $\mathrm{C} 8-\mathrm{C} 9$ & $1.357(3)$ & $\mathrm{N} 5-\mathrm{H} 5 \mathrm{~A}$ & $0.89(3)$ \\
\hline $\mathrm{C} 8-\mathrm{N} 5$ & $1.372(3)$ & $\mathrm{O} 8-\mathrm{H} 8 \mathrm{~A}$ & $0.82(4)$ \\
\hline $\mathrm{C} 8-\mathrm{H} 8$ & 0.9500 & $\mathrm{O} 8-\mathrm{H} 8 \mathrm{~B}$ & $0.88(4)$ \\
\hline $\mathrm{O} 1-\mathrm{C} 1-\mathrm{C} 2$ & $124.91(18)$ & $\mathrm{C} 8-\mathrm{C} 9-\mathrm{H} 9$ & 126.7 \\
\hline $\mathrm{O} 1-\mathrm{C} 1-\mathrm{C} 6$ & $124.28(17)$ & $\mathrm{N} 4-\mathrm{C} 9-\mathrm{H} 9$ & 126.7 \\
\hline $\mathrm{C} 2-\mathrm{C} 1-\mathrm{C} 6$ & $110.56(16)$ & $\mathrm{O} 2-\mathrm{N} 1-\mathrm{O} 3$ & $123.96(18)$ \\
\hline $\mathrm{C} 3-\mathrm{C} 2-\mathrm{C} 1$ & $125.53(17)$ & $\mathrm{O} 2-\mathrm{N} 1-\mathrm{C} 2$ & $118.23(17)$ \\
\hline $\mathrm{C} 3-\mathrm{C} 2-\mathrm{N} 1$ & $116.18(16)$ & $\mathrm{O} 3-\mathrm{N} 1-\mathrm{C} 2$ & $117.81(17)$ \\
\hline $\mathrm{C} 1-\mathrm{C} 2-\mathrm{N} 1$ & $118.28(17)$ & $\mathrm{O} 5-\mathrm{N} 2-\mathrm{O} 4$ & $122.65(17)$ \\
\hline $\mathrm{C} 2-\mathrm{C} 3-\mathrm{C} 4$ & $118.43(17)$ & $\mathrm{O} 5-\mathrm{N} 2-\mathrm{C} 4$ & $118.87(17)$ \\
\hline $\mathrm{C} 2-\mathrm{C} 3-\mathrm{H} 3$ & 120.8 & $\mathrm{O} 4-\mathrm{N} 2-\mathrm{C} 4$ & $118.47(16)$ \\
\hline $\mathrm{C} 4-\mathrm{C} 3-\mathrm{H} 3$ & 120.8 & $\mathrm{O} 6-\mathrm{N} 3-\mathrm{O} 7$ & $124.46(18)$ \\
\hline $\mathrm{C} 3-\mathrm{C} 4-\mathrm{C} 5$ & $121.33(17)$ & $\mathrm{O} 6-\mathrm{N} 3-\mathrm{C} 6$ & $117.85(18)$ \\
\hline $\mathrm{C} 3-\mathrm{C} 4-\mathrm{N} 2$ & $119.15(17)$ & $\mathrm{O} 7-\mathrm{N} 3-\mathrm{C} 6$ & $117.69(17)$ \\
\hline
\end{tabular}




\begin{tabular}{|c|c|c|c|}
\hline $\mathrm{C} 5-\mathrm{C} 4-\mathrm{N} 2$ & $119.50(17)$ & $\mathrm{N} 4-\mathrm{C} 7-\mathrm{N} 5$ & $108.37(19)$ \\
\hline $\mathrm{C} 6-\mathrm{C} 5-\mathrm{C} 4$ & $118.12(17)$ & $\mathrm{N} 4-\mathrm{C} 7-\mathrm{H} 7$ & 125.8 \\
\hline $\mathrm{C} 6-\mathrm{C} 5-\mathrm{H} 5$ & 120.9 & $\mathrm{~N} 5-\mathrm{C} 7-\mathrm{H} 7$ & 125.8 \\
\hline $\mathrm{C} 4-\mathrm{C} 5-\mathrm{H} 5$ & 120.9 & $\mathrm{C} 7-\mathrm{N} 4-\mathrm{C} 9$ & $108.85(19)$ \\
\hline $\mathrm{C} 5-\mathrm{C} 6-\mathrm{C} 1$ & $126.00(17)$ & $\mathrm{C} 7-\mathrm{N} 4-\mathrm{H} 4$ & $126.4(19)$ \\
\hline $\mathrm{C} 5-\mathrm{C} 6-\mathrm{N} 3$ & $116.53(17)$ & $\mathrm{C} 9-\mathrm{N} 4-\mathrm{H} 4$ & $124.7(19)$ \\
\hline $\mathrm{C} 1-\mathrm{C} 6-\mathrm{N} 3$ & $117.46(16)$ & $\mathrm{C} 7-\mathrm{N} 5-\mathrm{C} 8$ & $108.99(18)$ \\
\hline $\mathrm{C} 9-\mathrm{C} 8-\mathrm{N} 5$ & $107.10(19)$ & $\mathrm{C} 7-\mathrm{N} 5-\mathrm{H} 5 \mathrm{~A}$ & $123.3(18)$ \\
\hline $\mathrm{C} 9-\mathrm{C} 8-\mathrm{H} 8$ & 126.4 & $\mathrm{C} 8-\mathrm{N} 5-\mathrm{H} 5 \mathrm{~A}$ & $127.7(18)$ \\
\hline $\mathrm{N} 5-\mathrm{C} 8-\mathrm{H} 8$ & 126.4 & $\mathrm{H} 8 \mathrm{~A}-\mathrm{O} 8-\mathrm{H} 8 \mathrm{~B}$ & $102(3)$ \\
\hline $\mathrm{C} 8-\mathrm{C} 9-\mathrm{N} 4$ & $106.69(18)$ & & \\
\hline $\mathrm{O} 1-\mathrm{C} 1-\mathrm{C} 2-\mathrm{C} 3$ & $174.4(2)$ & $\mathrm{C} 3-\mathrm{C} 2-\mathrm{N} 1-\mathrm{O} 2$ & $-42.2(3)$ \\
\hline $\mathrm{C} 6-\mathrm{C} 1-\mathrm{C} 2-\mathrm{C} 3$ & $0.0(3)$ & $\mathrm{C} 1-\mathrm{C} 2-\mathrm{N} 1-\mathrm{O} 2$ & $136.7(2)$ \\
\hline $\mathrm{O} 1-\mathrm{C} 1-\mathrm{C} 2-\mathrm{N} 1$ & $-4.3(3)$ & $\mathrm{C} 3-\mathrm{C} 2-\mathrm{N} 1-\mathrm{O} 3$ & $137.2(2)$ \\
\hline $\mathrm{C} 6-\mathrm{C} 1-\mathrm{C} 2-\mathrm{N} 1$ & $-178.67(19)$ & $\mathrm{C} 1-\mathrm{C} 2-\mathrm{N} 1-\mathrm{O} 3$ & $-44.0(3)$ \\
\hline $\mathrm{C} 1-\mathrm{C} 2-\mathrm{C} 3-\mathrm{C} 4$ & $1.1(3)$ & $\mathrm{C} 3-\mathrm{C} 4-\mathrm{N} 2-\mathrm{O} 5$ & $-177.4(2)$ \\
\hline $\mathrm{N} 1-\mathrm{C} 2-\mathrm{C} 3-\mathrm{C} 4$ & $179.82(18)$ & $\mathrm{C} 5-\mathrm{C} 4-\mathrm{N} 2-\mathrm{O} 5$ & $1.2(3)$ \\
\hline $\mathrm{C} 2-\mathrm{C} 3-\mathrm{C} 4-\mathrm{C} 5$ & $-2.3(3)$ & $\mathrm{C} 3-\mathrm{C} 4-\mathrm{N} 2-\mathrm{O} 4$ & $1.5(3)$ \\
\hline $\mathrm{C} 2-\mathrm{C} 3-\mathrm{C} 4-\mathrm{N} 2$ & $176.28(19)$ & $\mathrm{C} 5-\mathrm{C} 4-\mathrm{N} 2-\mathrm{O} 4$ & $-179.89(19)$ \\
\hline $\mathrm{C} 3-\mathrm{C} 4-\mathrm{C} 5-\mathrm{C} 6$ & $2.3(3)$ & $\mathrm{C} 5-\mathrm{C} 6-\mathrm{N} 3-\mathrm{O} 6$ & $-132.1(2)$ \\
\hline $\mathrm{N} 2-\mathrm{C} 4-\mathrm{C} 5-\mathrm{C} 6$ & $-176.28(19)$ & $\mathrm{C} 1-\mathrm{C} 6-\mathrm{N} 3-\mathrm{O} 6$ & $49.1(3)$ \\
\hline $\mathrm{C} 4-\mathrm{C} 5-\mathrm{C} 6-\mathrm{C} 1$ & $-1.1(3)$ & $\mathrm{C} 5-\mathrm{C} 6-\mathrm{N} 3-\mathrm{O} 7$ & $47.3(3)$ \\
\hline $\mathrm{C} 4-\mathrm{C} 5-\mathrm{C} 6-\mathrm{N} 3$ & $-179.77(19)$ & $\mathrm{C} 1-\mathrm{C} 6-\mathrm{N} 3-\mathrm{O} 7$ & $-131.5(2)$ \\
\hline $\mathrm{O} 1-\mathrm{C} 1-\mathrm{C} 6-\mathrm{C} 5$ & $-174.5(2)$ & $\mathrm{N} 5-\mathrm{C} 7-\mathrm{N} 4-\mathrm{C} 9$ & $0.3(3)$ \\
\hline $\mathrm{C} 2-\mathrm{C} 1-\mathrm{C} 6-\mathrm{C} 5$ & $0.0(3)$ & $\mathrm{C} 8-\mathrm{C} 9-\mathrm{N} 4-\mathrm{C} 7$ & $0.0(3)$ \\
\hline $\mathrm{O} 1-\mathrm{C} 1-\mathrm{C} 6-\mathrm{N} 3$ & $4.2(3)$ & $\mathrm{N} 4-\mathrm{C} 7-\mathrm{N} 5-\mathrm{C} 8$ & $-0.6(3)$ \\
\hline $\mathrm{C} 2-\mathrm{C} 1-\mathrm{C} 6-\mathrm{N} 3$ & $178.65(18)$ & $\mathrm{C} 9-\mathrm{C} 8-\mathrm{N} 5-\mathrm{C} 7$ & $0.6(3)$ \\
\hline $\mathrm{N} 5-\mathrm{C} 8-\mathrm{C} 9-\mathrm{N} 4$ & $-0.3(3)$ & & \\
\hline
\end{tabular}

Hydrogen-bond geometry $\left(\AA,{ }^{\circ}\right)$

\begin{tabular}{|c|c|c|c|c|}
\hline$D-\mathrm{H} \cdots A$ & $D-\mathrm{H}$ & $\mathrm{H} \cdots A$ & $D^{\cdots} A$ & $D-\mathrm{H} \cdots A$ \\
\hline $\mathrm{N} 4-\mathrm{H} 4 \cdots \mathrm{O} 8^{\mathrm{i}}$ & $0.93(3)$ & $1.83(3)$ & $2.763(3)$ & $172(3)$ \\
\hline $\mathrm{N} 5-\mathrm{H} 5 A \cdots \mathrm{O} 1^{\mathrm{ii}}$ & $0.89(3)$ & $1.94(3)$ & $2.812(2)$ & $165(3)$ \\
\hline $\mathrm{N} 5-\mathrm{H} 5 A \cdots \mathrm{O} 3^{\mathrm{ii}}$ & $0.89(3)$ & $2.46(3)$ & $2.956(3)$ & $116(2)$ \\
\hline $\mathrm{O} 8-\mathrm{H} 8 A \cdots \mathrm{O} 4^{\mathrm{iii}}$ & $0.82(4)$ & $2.29(4)$ & $3.034(2)$ & $151(3)$ \\
\hline $\mathrm{O} 8-\mathrm{H} 8 B \cdots \mathrm{O} 1^{\mathrm{ii}}$ & $0.88(4)$ & $2.04(4)$ & $2.899(2)$ & $165(3)$ \\
\hline $\mathrm{O} 8-\mathrm{H} 8 B \cdots \mathrm{O} 6^{\mathrm{ii}}$ & $0.88(4)$ & $2.44(4)$ & $2.943(3)$ & $117(3)$ \\
\hline $\mathrm{C} 5-\mathrm{H} 5 \cdots \mathrm{O} 2^{\mathrm{iv}}$ & 0.95 & 2.49 & $3.383(3)$ & 157 \\
\hline $\mathrm{C} 7-\mathrm{H} 7 \cdots \mathrm{O}^{v}$ & 0.95 & 2.37 & $3.187(3)$ & 144 \\
\hline $\mathrm{C} 7-\mathrm{H} 7 \cdots \mathrm{O}^{3 i}$ & 0.95 & 2.47 & $2.955(3)$ & 112 \\
\hline $\mathrm{C} 8-\mathrm{H} 8 \cdots \mathrm{O} 4^{\mathrm{vi}}$ & 0.95 & 2.44 & $3.188(3)$ & 135 \\
\hline $\mathrm{C} 8-\mathrm{H} 8 \cdots \mathrm{O} 8$ & 0.95 & 2.53 & $3.255(3)$ & 133 \\
\hline $\mathrm{C} 9-\mathrm{H} 9 \cdots \mathrm{O} 7^{\mathrm{vii}}$ & 0.95 & 2.48 & $3.349(3)$ & 152 \\
\hline
\end{tabular}

Symmetry codes: (i) $-x+1,-y+1, z+1 / 2$; (ii) $x, y+1, z$; (iii) $x-1 / 2,-y+3 / 2, z$; (iv) $-x+3 / 2, y+1 / 2, z-1 / 2$; (v) $-x+3 / 2, y+1 / 2, z+1 / 2$; (vi) $x-1 / 2,-y+1 / 2, z$; (vii) $-x+1,-y, z+1 / 2$. 